\title{
CARACTERÍSTICAS CLÍNICAS DE LA HEPATOTOXICIDAD ASOCIADA A LA PIRAZINAMIDA EN PACIENTES DE UN HOSPITAL DE LIMA, PERÚ
}

\author{
Teodoro Oscanoa (10 1,2,a,c, Saul Moscol (10) ${ }^{3, \mathrm{~b}}$, José Amado (10) $2, \mathrm{c}$ \\ 1 Centro de Investigación de Seguridad de Medicamentos, Facultad de Medicina Humana, Universidad de San Martín \\ de Porres, Lima, Perú. \\ 2 Facultad de Medicina de la Universidad Nacional Mayor de San Marcos, Lima, Perú. \\ 3 Servicio de Neumología, Hospital Nacional Guillermo Almenara Irigoyen, Lima, Perú. \\ a Médico Internista; ${ }^{\mathrm{b}}$ Médico Neumólogo; ${ }^{\mathrm{c}}$ doctor en Medicina.
}

\section{RESUMEN}

Con el objetivo de determinar las características de la enfermedad hepática inducida por el medicamento (DILI) se realizó un estudio de pacientes adultos con diagnóstico de tuberculosis y esquema de tratamiento antituberculoso con pirazinamida. El análisis de causa efecto de la DILI fue mediante el proceso de reexposición. Se encontraron 10 pacientes con DILI asociada a pirazinamida, la mediana de edad y de estancia hospitalaria fue de 40,5 años (rango 22-76) y 41 días (rango 11-130), respectivamente. La mediana de presentación del evento fue de 14 días (rango 3-46), 4 pacientes presentaron ictericia, 5 tuvieron patrón hepatocelular, 3 mixtas y 2 colestásicos. La presentación de la DILI fue leve en 6 casos (60\%) y moderados en 3 (30\%). En conclusión, la DILI asociada a la pirazinamida requiere estancia hospitalaria prolongada, se presenta con ictericia en un poco más de un tercio de los casos siendo el patrón predominante el hepatocelular.

Palabras clave: Tuberculosis; Hepatotoxicidad; Antituberculosos; Pirazinamida; Reacción Adversa Medicamentosa; Estancia Hospitalaria. (Fuente: DeCS BIREME)

\section{CLINICAL CHARACTERISTICS OF PYRAZINAMIDE- ASSOCIATED HEPATOTOXICITY IN PATIENTS AT A HOSPITAL IN LIMA, PERU}

\begin{abstract}
In order to determine the characteristics of drug-induced liver injury (DILI), adult patients diagnosed with tuberculosis and with an anti-tuberculosis treatment scheme including pyrazinamide were studied. The re-exposure process was used for the cause-effect analysis of the DILI. A total of 10 patients were found with pyrazinamide-associated DILI; the median age and hospital stay were 40.5 years (from 22 to 76 years) and 41 days (from 11 to 130 days), respectively. The median time in which the events appeared was 14 days (from 3 to 46 days); jaundice was observed in 4 patients and radiological patterns such as hepatocellular, mixed and cholestatic were found in 5, 3 and 2 patients, respectively. Mild presentation of DILI was observed in 6 cases $(60 \%)$ and moderate in $3(30 \%)$. In conclusion, pyrazinamide-associated DILI required prolonged hospital stay, presented jaundice in little more than a third of the cases, and radiologically, the hepatocellular pattern predominated.
\end{abstract}

Keywords: Tuberculosis; Drug Induced Liver Injury; Antituberculosis Drugs; Pyrazinamide; Adverse Drug Reaction; Length of Stay. (Source: MeSH NLM).

Citar como: Oscanoa T, Moscol S, Amado J. Características clínicas de la hepatotoxicidad asociada a la pirazinamida en pacientes de un hospital de Lima, Perú. Rev Peru Med Exp Salud Publica. 2020;37(3):51620. doi: https://doi.org/10.17843/ rpmesp.2020.373.4684.

Correspondencia: Teodoro Oscanoa tjoscanoae@gmail.com

Recibido: $20 / 07 / 2019$ Aprobado: $24 / 06 / 2020$ En línea: 02/09/2020

\section{INTRODUCCIÓN}

La tuberculosis es un problema de salud pública en el Perú y la tasa de incidencia de morbilidad total es de 99,5 por 100000 habitantes ${ }^{(1)}$. Las dos grandes dificultades con el tratamiento son la drogorresistencia y las reacciones adversas medicamentosas (RAM). La frecuencia de RAM a fármacos de primera línea (isoniazida, rifampicina, pirazinamida y etambutol) es de $3,4 \%$, la más grave es la enfermedad hepática inducida por medicamentos (DILI, por sus siglas en inglés), que tiene una incidencia del 2 al $28 \%$ según el régimen terapéutico y las características de los pacientes (edad, raza y sexo) ${ }^{(2)}$.

En el tratamiento de la tuberculosis se emplean cuatro antibióticos, tres de los cuales se administran de manera simultánea y son potencialmente hepatotóxicos: la isoniazida $(\mathrm{H})$, la 
rifampicina (R) y la pirazinamida $(\mathrm{Z})$. Cuando se analiza la causalidad de la relación entre los antituberculosos administrados y la DILI, generalmente se establece que los tres fármacos estarían asociados, puesto que la administración fue simultánea. El instrumento más utilizado para establecer la relación causa-efecto de un medicamento y la DILI es el método de evaluación de causalidad de Roussel Uclaf (RUCAM, por sus siglas en inglés) ${ }^{(3)}$, el cual consiste en una tarjeta de puntuación algorítmica que permite determinar si la lesión es hepatocelular, colestática o mixta; sin embargo, cuando un paciente recibe más de un fármaco potencialmente hepatotóxico, como sucede en el tratamiento de tuberculosis, el método recomienda considerar estos tres fármacos como uno solo ${ }^{(4)}$, por lo tanto es difícil establecer si la DILI estuvo asociada exclusivamente a pirazinamida.

Actualmente existe un vacío de conocimiento sobre las características o modelos fenotípicos de la DILI asociada a pirazinamida. Los casos reportados en el Perú tienden a englobar los tres fármacos $(\mathrm{H}, \mathrm{R}$ y $\mathrm{Z})$ con la DILI sin especificar si fue posible identificar a la pirazinamida ${ }^{(5)}$. El objetivo del presente estudio es describir las características clínicas y de laboratorio de la DILI inducida por pirazinamida utilizando el criterio del RUCAM.

\section{EL ESTUDIO}

\section{Diseño y ámbito de estudio}

El presente es un estudio observacional y retrospectivo. Se revisaron las historias clínicas de pacientes hospitalizados con diagnóstico de DILI inducida por medicamentos antituberculosos, desde enero de 2014 hasta enero de 2019, en el Hospital Almenara, de Lima, Perú, hospital de alta complejidad y de referencia nacional.

\section{Criterios de inclusión}

Los criterios de inclusión fueron tener una edad de $\geq 18$ años, diagnóstico de tuberculosis por baciloscopia y/o cultivo y uso de pirazinamida como parte del tratamiento. En todos los pacientes se verificó la existencia del consentimiento informado firmado del tratamiento antituberculoso.

\section{Procedimiento para identificar la DILI por pirazinamida}

Se usaron los criterios de DILI Expert Working Group ${ }^{(4)}$, los cuales consisten en la presencia de uno de los siguientes hallazgos: niveles de alanina aminotransferasa (ALT) igual o cinco veces por encima del límite superior normal (LSN); fosfatasa alcalina (FA) igual o mayor a dos veces por encima del LSN (especialmente si se acompaña de elevación en la concentración de 5'-nucleotidasa o gammaglutamil transpeptidasa (GGT), en ausencia de patología ósea que se conozca que incrementa la fosfatasa alcalina); o elevación igual o mayor al triple del LSN de la concentración de ALT y elevación simultánea de la concentración de bilirrubina superior al LSN ${ }^{(4)}$. Para determinar la severidad se consideró leve

\section{MENSAJES CLAVE}

\begin{abstract}
Motivación para realizar el estudio: La hepatotoxicidad inducida por fármacos antituberculosos es un problema en el tratamiento, se conoce las reacciones adversas relacionadas a la rifampicina e isoniacida, sin embargo, hace faltan estudios sobre la pirazinamida específicamente.
\end{abstract}

Principales hallazgos: El presente estudio muestra que la hepatotoxicidad inducida por pirazinamida inicia a la tercera semana de exposición, se presenta ictericia en un tercio de los casos y el patrón predominante es el hepatocelular.

Implicancias: El estudio y la fenotipificación adecuada de la hepatotoxicidad inducida por pirazinamida permitiría, en el futuro, su prevención mediante estudios farmacogenéticos.

si la bilirrubina era $<2 \mathrm{mg} / \mathrm{dl}$, moderada cuando la bilirrubina era $\geq 2 \mathrm{mg} / \mathrm{dl}$ o se presenta con síntomas vinculados a la hepatitis, si la bilirrubina $\geq 2 \mathrm{mg} / \mathrm{dl}$ agregando uno de los siguientes criterios: razón normalizada internacional (INR) $\geq 1,5$, ascitis, encefalopatía, o afección de otro órgano y muerte o trasplante cuando estuvo relacionando a la DILI ${ }^{(4)}$.

Con el objetivo de identificar la asociación con pirazinamida, se verificó en las historias clínicas la descripción del proceso en cuatro fases: exposición, retiro de fármacos, reexposición ${ }^{(6)}$ y evolución (seguimiento). La fase de exposición fue de administración de los fármacos (por ejemplo, en el esquema para tuberculosis sensible, rifampicina, isoniacida, etambutol y pirazinamida) e identificación de los criterios de la DILI. Una fase consistió en el retiro de todos los fármacos, hasta conseguir la normalización de las enzimas hepáticas. La fase de reexposición es donde progresivamente se vuelve a administrar los medicamentos uno a uno, generalmente comenzando por etambutol luego rifampicina, sigue isoniazida y finalmente pirazinamida. El segundo método utilizado para determinar la asociación especifica de la DILI a pirazinamida fue la verificación de la suspensión única y exclusiva de pirazinamida y no la observación de criterios de la DILI en la evolución y seguimiento del paciente.

\section{Variables de estudio}

En la revisión de las historias clínicas se extrajeron datos como edad, sexo, consumo de alcohol y cigarrillos, peso y talla. Para identificar las características de la DILI se incluyeron síntomas, signos, número de días de hospitalización, niveles de ALT, FA, GGT. Se verificó el registro del proceso de reexposición a pirazinamida y el tratamiento antituberculoso al alta del paciente.

\section{Análisis estadístico}

Se analizaron las variables en frecuencias usando porcentajes, mediana y rango. Para obtener el número de veces sobre 
el LSN que estaba aumentada el ALT y la FA de los casos reportados, se dividió el valor sérico obtenido del paciente con el LSN.

\section{Aspectos éticos}

El presente estudio fue aprobado por el Comité de Ética del Hospital Almenara. Se establecieron las estrategias necesarias para mantener la privacidad de la información de los pacientes.

\section{HALLAZGOS}

\section{Características de los pacientes}

Durante el periodo del estudio, 507 casos de tuberculosis fueron internados en el Hospital Almenara, de los cuales $10(1,9 \%)$ fueron por DILI asociados a pirazinamida (Tabla 1 y 2 ). La mediana de días de hospitalización por DILI asociados a pirazinamida fue de 41 (rango 11-130). El diagnóstico fue tuberculosis pulmonar, pleural y multisistémica en 7,2 y 1 casos, respectivamente. La mediana de edad fue de 40,5 años. La evolución fue favorable en todos los pacientes.

\section{Características de la DILI}

Siete pacientes fueron diagnosticados con DILI con el criterio de ALT $\geq 5$ veces por encima del LSN. Con FA $\geq 2$ veces

Tabla 1. Características generales de los pacientes con tuberculosis con enfermedad hepática inducida por pirazinamida $(\mathrm{Z})$

\begin{tabular}{lc}
\hline Características & Valor \\
\hline Mediana de edad (rango) & $40,5(22-76)$ \\
Sexo masculino, n/N (\%) & $5 / 10(50 \%)$ \\
Consumo de alcohol, n/N (\%) & $3 / 10(30 \%)$ \\
Consumo de cigarrillos, n/N (\%) & $2 / 10(20 \%)$ \\
Mediana de índice de masa corporal (rango) & $21,8(16,1-26,5)$ \\
Mediana de días hospitalización (rango) & $41(11-130)$ \\
Ictericia, n/N (\%) & $4 / 10(40 \%)$ \\
Criterios de la DILI & \\
ALT (5 veces sobre LSN), mediana (rango) & $6,85(1,4-43,4)$ \\
FA (>2 veces sobre LSN), mediana (rango) & $2,15(1-4,4)$ \\
Bilirrubina total (mg/dL), mediana (rango) & $1,1(0,5-9,5)$, \\
GGT (veces sobre LSN), mediana (rango) & $4,52(1,1-13,55)$ \\
RUCAM & \\
Hepatocelular, n/N (\%) & $5 / 10(50 \%)$ \\
Colestático, n/N (\%) & $2 / 10(20 \%)$ \\
Mixto, n/N (\%) & $3 / 10(30 \%)$ \\
Análisis de causalidad* & \\
Definitiva o muy probable, n/N & $4 / 10(40 \%)$ \\
Probable, n/N (\%) & $6 / 10(60 \%)$ \\
Fase de reexposición a Z, n/N (\%) & $8 / 10(80 \%)$ \\
\hline
\end{tabular}

ALT: alanina aminotransferasa, FA: fosfatasa alcalina; GGT: gammaglutamil transpeptidasa; LSN: límite superior normal, RUCAM: método de evaluación de causalidad de Roussel Uclaf.

* RUCAM instrumento de atribución de causalidad con puntaje desde, excluido (puntaje $<1$ ), improbable (1-2), posible (3-5), probable (6-8) y altamente probable $(>8)$. por encima del LSN (especialmente si se acompaña de elevación en la concentración de GGT, en ausencia de patología ósea que se conozca que incrementa la fosfatasa alcalina), en tres pacientes. Adicionalmente, cuatro pacientes presentaron elevación mayor o igual al triple de la concentración de ALT y elevación simultánea de la concentración de bilirrubina superior a 2 veces el LSN (Tabla 1 y 2 ).

La mediana de presentación del evento fue de 14 días (rango 3-46). El 40\% de pacientes presentó ictericia; y dos pacientes (20\%) erupción cutánea y prurito. Según los criterios de RUCAM, el patrón de lesión en 5 pacientes $(50 \%)$ fue hepatocelular, 3 (30\%) mixtas y 2 (20\%) colestásicos. La media y desviación estándar de la FA y ALT fue de 2,11 $(0,93)$ y $12,24(12,7)$ veces el LSN. La media y desviación estándar de bilirrubina total fue de $2,51 \mathrm{mg} / \mathrm{dL}(3,05)$. Una paciente desarrolló la DILI durante la gestación. Las comorbilidades asociadas fueron infección por VIH (1), diabetes mellitus (1). La presentación de la DILI fue leve en 6 (60\%), moderada en $3(30 \%)$ y severa en $1(10 \%)$.

\section{Causalidad de la DILI con pirazinamida}

La asociación de la DILI con pirazinamida se hizo en 8 pacientes mediante el proceso reexposición; mientras que en dos pacientes porque se suspendió únicamente pirazinamida y se verificó la remisión del evento. Ocho pacientes con tuberculosis, pansensibles al momento de la DILI, estuvieron tomando rifampicina, isoniacida, etambutol y pirazinamida y fueron referidos y hospitalizados por DILI, durante la hospitalización se realizó el proceso de reexposición el cual identificó a pirazinamida como asociado a DILI y descartó a isoniacida y rifampicina. En la paciente gestante la reexposición se realizó durante el periodo de puerperio, el cual identificó a pirazinamida como asociado a DILI y continuó tratamiento con rifampicina, isoniacida y etambutol (Tabla 1).

\section{DISCUSIÓN}

El presente estudio sobre las características de la DILI asociado a $\mathrm{Z}$ encontró que se presenta con más frecuencia en la tercera semana de administración, el $40 \%$ de los casos presentó ictericia, siendo el patrón de lesión predominante el hepatocelular. El grado de severidad de la DILI más frecuente fue leve y moderado. El 1,97\% fue hospitalizado por esta causa con una mediana de 41 días.

Los hallazgos del presente estudio pueden compararse con dos estudios previos. Abbara et al. ${ }^{(7)}$ encontraron que más de la mitad de los casos ocurre en las primeras dos semanas y la frecuencia de ictericia es del $12 \%$ de los casos; usaron los criterios de la DILI y el RUCAM como instrumento de causalidad; describieron las características clínicas y bioquímicas de 105 pacientes con DILI a fármacos antituberculosos (rifampicina, isoniacida y pirazinamida); sin embargo, no se realizó el proceso de reintroducción de pirazi- 
Tabla 2. Características específicas de los pacientes con tuberculosis con enfermedad hepática inducida por pirazinamida.

\begin{tabular}{|c|c|c|c|c|c|c|c|c|c|c|c|c|}
\hline \multirow[b]{2}{*}{ Caso } & \multirow[b]{2}{*}{$\begin{array}{l}\text { Sexo/ } \\
\text { edad }\end{array}$} & \multirow[b]{2}{*}{ Diagnóstico } & \multirow[b]{2}{*}{$\begin{array}{l}\text { Fármacos al } \\
\text { momento de } \\
\text { la DILI }\end{array}$} & \multicolumn{5}{|c|}{ Característica DILI } & \multicolumn{2}{|c|}{ RUCAM } & \multirow[b]{2}{*}{$\begin{array}{l}\text { Procesode } \\
\text { reexposición } \\
\text { a Z }\end{array}$} & \multirow[b]{2}{*}{$\begin{array}{c}\text { Tratamiento al alta hos- } \\
\text { pitalaria }\end{array}$} \\
\hline & & & & Ictericia & $\begin{array}{c}\text { ALT } \\
\text { (veces } \\
\text { aumentada } \\
\text { sobre LSN) }\end{array}$ & $\begin{array}{c}\text { FA } \\
\text { (veces } \\
\text { aumentada } \\
\text { sobre LSN) }\end{array}$ & $\begin{array}{c}\text { Bilirrubina } \\
\text { total } \\
(\mathbf{m g} / \mathbf{d L})\end{array}$ & $\begin{array}{c}\text { GGT } \\
\text { (veces } \\
\text { aumentada } \\
\text { sobre LSN) }\end{array}$ & Patrón & $\begin{array}{l}\text { Análisis de } \\
\text { causalidad }^{*}\end{array}$ & & \\
\hline 1 & $F / 41$ & $\begin{array}{l}\text { TB pulmonar } \\
\text { resistente a } \mathrm{H}\end{array}$ & $\mathrm{R}, \mathrm{H}, \mathrm{E}, \mathrm{Z}$ & Sí & 14,1 & 2,01 & 6,4 & 12,1 & $\begin{array}{l}\text { Hepato-Ce- } \\
\text { lular }\end{array}$ & $\begin{array}{l}\text { Definitiva o } \\
\text { muy pro- } \\
\text { bable }\end{array}$ & Sí & $\begin{array}{c}\text { Levofloxacina-kanamicina, } \\
\text { cicloserina, E, R }\end{array}$ \\
\hline 2 & $\mathrm{M} / 69$ & $\begin{array}{l}\text { TB pulmonar } \\
\text { pansensible }\end{array}$ & $\mathrm{R}, \mathrm{H}, \mathrm{E}, \mathrm{Z}$ & No & 19,2 & 2,0 & 0,5 & 5,2 & $\begin{array}{l}\text { Hepato-Ce- } \\
\text { lular }\end{array}$ & Probable & Sí & Levofloxacina, E, H, R \\
\hline 3 & $\mathrm{~F} / 59$ & $\begin{array}{l}\text { TB pulmonar } \\
\text { pansensible }\end{array}$ & $\mathrm{R}, \mathrm{H}, \mathrm{E}, \mathrm{Z}$ & Sí & 43,4 & 1,6 & 9,5 & 13,5 & $\begin{array}{l}\text { Hepato-Ce- } \\
\text { lular }\end{array}$ & Probable & Sí & E, R, H, levofloxacina \\
\hline 4 & $\mathrm{M} / 64$ & $\begin{array}{l}\text { TB pulmonar } \\
\text { resistente a } \\
\mathrm{H}, \mathrm{R}, \mathrm{E}, \mathrm{Z}\end{array}$ & $\begin{array}{l}\text { Kanamicina, } \\
\text { levofloxacina, } \\
\text { etionamida, } \\
\text { cicloserica y Z }\end{array}$ & No & 1,4 & 4,4 & 0,5 & 7,3 & $\begin{array}{l}\text { Colestá-Ti- } \\
\text { co }\end{array}$ & Probable & $\mathrm{No}^{* *}$ & $\begin{array}{c}\text { Amikacina, levofloxacina, } \\
\text { imipenem/cilasta-tina,etio- } \\
\text { namida y amoxicilina/ácido } \\
\text { clavulánico }\end{array}$ \\
\hline 5 & $\mathrm{~F} / 40$ & $\begin{array}{l}\text { TB pulmonar } \\
\text { resistente } \mathrm{H}, \mathrm{R}, \mathrm{E} \text {, } \\
\text { estretomicina y } \\
\text { etionamida }\end{array}$ & $\begin{array}{l}\text { Kanamicina, } \\
\text { levofloxa- } \\
\text { cina, Z, E, } \\
\text { etionamida, } \\
\text { cicloserina }\end{array}$ & No & 19,1 & 1,8 & 0,9 & 4,6 & $\begin{array}{l}\text { Hepato-Ce- } \\
\text { lular }\end{array}$ & Probable & Sí & $\begin{array}{l}\text { Kanamicina, lefofloxacina, } \\
\text { e, cicloserica, ácido paraa- } \\
\text { minosalicílico, amoxicilina/ } \\
\text { ácido clavulánico }\end{array}$ \\
\hline 6 & $\mathrm{M} / 22$ & $\begin{array}{l}\text { TB multisistémi- } \\
\text { ca pansensible }\end{array}$ & $\mathrm{R}, \mathrm{H}, \mathrm{E}, \mathrm{Z}$ & No & 1,6 & 2,7 & 0,7 & 4,5 & $\begin{array}{l}\text { Colestá-Ti- } \\
\text { co }\end{array}$ & Probable & Sí & $\mathrm{R}, \mathrm{H}, \mathrm{E}$, levofloxacino \\
\hline 7 & $\mathrm{M} / 76$ & $\begin{array}{l}\text { TB pleural } \\
\text { pansensible }\end{array}$ & $\mathrm{R}, \mathrm{H}, \mathrm{E}, \mathrm{Z}$ & Sí & 5,7 & 2,2 & 2,5 & 3,4 & Mixto & Probable & Sí & R, H y levofloxacino \\
\hline 8 & $\mathrm{~F} / 34$ & $\begin{array}{l}\text { TB pulmonar } \\
\text { pansensible }\end{array}$ & $\mathrm{R}, \mathrm{H}, \mathrm{E}, \mathrm{Z}$ & Sí & 4,9 & 2,1 & 2,4 & 4,3 & Mixto & $\begin{array}{l}\text { Definitiva o } \\
\text { muy pro- } \\
\text { bable }\end{array}$ & Sí & $\begin{array}{c}\text { Kanamicina, ciprofloxacina, } \\
\text { cicloserica, } \\
\text { etinamida }\end{array}$ \\
\hline 9 & $\mathrm{~F} / 36$ & $\begin{array}{l}\text { TB pulmonar } \\
\text { pansensible / } \\
\text { gestante }\end{array}$ & $\mathrm{R}, \mathrm{H}, \mathrm{E}, \mathrm{Z}$ & No & 8,0 & 1,31 & 0,6 & 1,1 & $\begin{array}{l}\text { Hepato-Ce- } \\
\text { lular }\end{array}$ & $\begin{array}{l}\text { Definitiva o } \\
\text { muy pro- } \\
\text { bable }\end{array}$ & Sí & $\mathrm{R}, \mathrm{H}, \mathrm{E}$, levofloxacina \\
\hline 10 & $\mathrm{M} / 33$ & $\begin{array}{l}\text { TB pleural } \\
\text { pansensible }\end{array}$ & $\mathrm{R}, \mathrm{H}, \mathrm{E}, \mathrm{Z}$ & No & 5,0 & 1,0 & 1,1 & 1,3 & Mixto & $\begin{array}{l}\text { Definitiva o } \\
\text { muy pro- } \\
\text { bable }\end{array}$ & $\mathrm{No}^{* *}$ & $\mathrm{R}, \mathrm{H}, \mathrm{E}$ \\
\hline
\end{tabular}

F: femenino; M: masculino; TB: tuberculosis; DILI: enfermedad hepática inducida por medicamento; RUCAM: método de evaluación de causalidad de Roussel Uclaf; Z: pirazinamida; R: rifampicina; H: isoniacida; E: etambutol; ALT: alanina aminotransferasa; FA: fosfatasa alcalina; GGT: gammaglutamil transpeptidasa; LSN: límite superior normal.

${ }^{*}$ RUCAM: instrumento de atribución de causalidad con puntaje desde, excluido (puntaje $<1$ ), improbable (1-2), posible (3-5), probable (6-8) y altamente probable (>8); ${ }^{* *}$ remite DILI con suspensión de Z.

namida. An et al. encontraron mayor frecuencia en mujeres, $21 \%$ tuvo ictericia y en el $75 \%$ ocurrió antes de los 2 meses y que fue más frecuente en los que recibieron el esquema con pirazinamida; el reporte no describió el instrumento de causalidad utilizado ${ }^{(8)}$, ni separó sus hallazgos del DILI inducido por $\mathrm{Z}$.

La fenotipificación de la DILI asociada a pirazinamida se estudia mediante dos estrategias; con esquemas que excluyen rifampicina e isoniacida; y otros investigan el uso de pirazinamida juntamente con rifampicina e isoniacida comparado con aquellos que recibieron solo rifampicina e isoniacida. Younossian et al. estudiaron pacientes con tuberculosis latente, donde se utilizó pirazinamida y etambutol, el cual fue discontinuado en $58 \%$ de los pacientes a los 119 días por hepatotoxicidad, elevación de enzimas hepáticas más de 4 veces el valor normal y síntomas gastrointestinales ${ }^{(9)}$. Be- dini et al. estudiaron pacientes con tuberculosis latente, tratados con $\mathrm{Z}$ y levofloxacina, el $41 \%$ de los pacientes hizo DILI, con un incremento mayor de 4 veces de AST y ALT ${ }^{(10)}$. Chang et al. compararon los esquemas de tratamiento de pirazinamida, rifampicina e isoniacida, con aquellos que recibieron solo rifampicina e isoniacida; el estudio encontró que la DILI en el grupo con pirazinamida y sin pirazinamida fue $2,6 \%$ y $0,8 \%$, respectivamente ${ }^{(11)}$; el estudio descrito, utilizó como criterio de hepatotoxicidad la elevación de ALT en más de 3 veces el LSN ${ }^{(11)}$. Un metaanálisis comparó el riesgo de hepatotoxicidad del esquema de pirazinamida y rifampicina comparado con solo isoniacida y encontró que el esquema con pirazinamida no aumentó el riesgo de hepatotoxicidad, los criterios de hepatotoxicidad fue ALT igual o más de 3 veces el LSN ${ }^{(12)}$.

La DILI asociada a pirazinamida representa cerca del $2 \%$ de las causas de internamiento en pacientes diagnosticados 
con tuberculosis, pero requieren más de 45 días de hospitalización. En el mundo, la DILI asociada a antituberculosos y/o la multidrogorresistencia es una de las principales causas de estancia hospitalizaria prolongada ${ }^{(13-16)}$.

Las limitaciones del presente estudio están relacionadas al diseño retrospectivo, que restringen el registro de datos clínicos y de laboratorios. Otro problema fue la falta de una estandarización de las definiciones operacionales para la fenotipificación de la DILI asociada a antituberculosos. Las revisiones de Tostmann et al. y Hosford et al. ${ }^{(17)}$ incluyen hasta 5 y 8 definiciones operacionales de DILI ${ }^{(2)}$, respectivamente. Otra limitación fue que el RUCAM no está diseñado para las DILI crónicas ni para la coexistencia de enfermedades hepáticas preexistentes ${ }^{(18)}$.

\section{REFERENCIAS BIBLIOGRÁFICAS}

1. Alarcón V, Alarcón E, Figueroa C, Mendoza-Ticona A. Tuberculosis en el Perú: situación epidemiológica, avances y desafíos para su control. Rev Peru Med Exp Salud Publica. 2017;34(2):299-310. doi: 10.17843/rpmesp.2017.342.2384.

2. Tostmann A, Boeree MJ, Aarnoutse RE, de Lange WC, van der Ven AJ, Dekhuijzen R. Antituberculosis drug-induced hepatotoxicity: concise up-to-date review. J Gastroenterol Hepatol. 2008;23(2):192-202. doi: 10.1111/j.1440-1746.2007.05207.x.

3. Danan G, Teschke R. RUCAM in Drug and Herb Induced Liver Injury: The Update. Int J Mol Sci. 2015;17(1):14. doi: 10.3390/ijms17010014.

4. Aithal GP, Watkins PB, Andrade RJ, Larrey D, Molokhia M, Takikawa, et al. Case definition and phenotype standardization in drug-induced liver injury. Clin Pharmacol Ther. 2011;89(6):806-815. doi: 10.1038/ clpt.2011.58.

5. Reto Valiente LV, Castillo Vergara J, Pichilingue Reto P, Pichilingue Prieto OA. Hepatotoxicidad por Fármacos Antituberculosos en Pediatria. Rev Gastroenterol Peru. 2005;25(4):362-365.

6. Sharma SK, Singla R, Sarda P, Mohan A, Makharia G, Jayaswal A, et al. Safety of 3 different reintroduction regimens of antituberculosis drugs after development of antituberculosis treatment-induced hepatotoxicity. Clin Infect Dis. 2010;50(6):833-839. doi: 10.1086/650576.

7. Abbara A, Chitty S, Roe JK, Ghani R, Collin SM, Ritchie A, et al. Drug-induced liver injury from antituberculous treatment: a retrospective study from a large TB centre in the UK. BMC Infect Dis. 2017;17(1):231. doi: 10.1186/s12879-017-2330-z.

8. An H, Wu X, Wang Z, Xu J, Zheng S, Wang K. The clinical characteristics of anti-tuberculosis drug induced liver injury in 2457 hospitalized patients with tuberculosis in China. Afr J Pharm Pharmacol. 2013;7(13):710-714.

9. Younossian AB, Rochat T, Ketterer JP, Wacker J, Janssens JP. High hepatotoxicity of pyrazinamide and ethambutol for treatment of latent tuberculosis. Eur Respir J. 2005;26(3):462-464. doi: 10.1183/09031936.05.00006205.
En conclusión, la DILI asociada a pirazinamida se inicia a la tercera semana de exposición, presenta ictericia en más de un tercio de los casos, predomina el patrón hepatocelular y tiene estancia hospitalaria prolongada.

Agradecimientos: Al jefe, a los médicos asistentes, médicos residentes y personal en general del Servicio de Neumología del Hospital Almenara, ESSALUD, de Lima, Perú.

Contribuciones de autoría: TO ha concebido el artículo, analizado los datos, redactado y aprobado la versión final del manuscrito. SM ha aportado con información sobre pacientes y recolección de datos. JA se encargó de analizar datos y realizar una revisión crítica del artículo.

Conflictos de interés: Los autores declaran no tener conflicto de interés.

10. Bedini A, Garlassi E, Stentarelli C, Petrella S, Meacci M, Meccugni B, et al. Multidrug-resistant tuberculosis outbreak in an Italian prison: tolerance of pyrazinamide plus levofloxacin prophylaxis and serial interferon gamma release assays. New Microbes New Infect. 2016;12:45-51. doi: 10.1016/j. nmni.2016.03.010.

11. Chang KC, Leung CC, Yew WW, Lau TY, Tam CM. Hepatotoxicity of pyrazinamide: cohort and case-control analyses. Am J Respir Crit Care Med. 2008;177(12):1391-1396. doi: 10.1164/rccm.200802-355OC.

12. Camacho A, Pérez-Camacho I, Rivero A, Natera C, Garcia-Lazaro M, Caston J, et al. Use of rifampicin plus pyrazinamide for antituberculosis prophylaxis does not increase the risk of severe hepatotoxicity in HIV patients: meta-analysis of randomized controlled clinical trials. Enferm Infecc Microbiol Clin. 2010;28(4):239-244. doi: 10.1016/j.eimc.2009.04.003.

13. Chan ED, Laurel V, Strand MJ, Chan JF, Huynh ML, Goble M, et al. Treatment and outcome analysis of 205 patients with multidrug-resistant tuberculosis. Am J Respir Crit Care Med. 2004;169(10):1103-1109. doi: 10.1164/rccm.200308-1159OC.

14. Leimane V, Riekstina V, Holtz TH, Zarovska E, Skripconoka V, Thorpe LE, et al. Clinical outcome of individualised treatment of multidrug-resistant tuberculosis in Latvia: a retrospective cohort study. Lancet. 2005;365(9456):318-326. doi: 10.1016/S0140-6736(05)17786-1.

15. Dheda K, Migliori GB. The global rise of extensively drug-resistant tuberculosis: is the time to bring back sanatoria now overdue? Lancet. 2012;379(9817):773-775. doi: 10.1016/S0140-6736(11)61062-3.

16. Evans G. Bad news in the global village: are U.S. hospitals ready for the XDR-TB strain? World Hosp Health Serv. 2007;43(3):46-48.

17. Hosford JD, von Fricken ME, Lauzardo M, Chang M, Dai Y, Lyon JA, et al. Hepatotoxicity from antituberculous therapy in the elderly: a systematic review. Tuberculosis (Edinb). 2015;95(2):112-122. doi: 10.1016/j. tube.2014.10.006.

18. Teschke R, Schulze J, Eickhoff A, Danan G. Drug Induced Liver Injury: Can Biomarkers Assist RUCAM in Causality Assessment? Int J Mol Sci. 2017;18(4):803. doi: 10.3390/ijms18040803. 